\title{
Expression of the Cancer Stem Cell Markers CD44 and CD133 in Colorectal Cancer: An Immunohistochemical Staining Analysis
}

\author{
Injae Hong, Seong Woo Hong, Yeo Goo Chang, Woo Yong Lee, Byungmo Lee, Yun Kyung Kang ${ }^{1}$ \\ You Sun $\mathrm{Kim}^{2}$, In Wook Paik, Hyucksang Lee \\ Departments of Surgery, ${ }^{1}$ Pathology, and ${ }^{2}$ Internal Medicine, Inje University Seoul Paik Hospital, Seoul, Korea
}

Purpose: The aim of this study was to assess the expressions of CD44 and CD133 in colorectal cancer tissue by using immunohistochemical staining and to analyze the clinical significance of the expressions related to other clinicopathological data and survival results.

Methods: One hundred sixty-two patients with a biopsy-proven colorectal adenocarcinoma who were operated on between January 1998 and August 2004 were enrolled in this study. Immunohistochemical staining for CD44 and CD133 was performed on primary colorectal cancer tissue, metastatic lymph nodes, and synchronous and metachronous metastatic tumor tissues if available.

Results: CD44 expression was stronger in the primary tumor than in metastatic lymph nodes $(\mathrm{P}<0.001)$, and CD133 expression tended to be stronger in primary tumor than in metastatic lymph nodes $(\mathrm{P}=0.057)$. No significant correlation was found between the CD44 and the CD133 expressions. The cases with recurrence showed low expression of CD44 $(\mathrm{P}=$ 0.017). CD133 expression was lower in cases with elevated CA $19-9$ serum levels $(\mathrm{P}=0.028)$ and advanced $\mathrm{T}$ stage $(\mathrm{P}=$ 0.038 . Multivariate analysis proved that low expression of CD44 was an independent prognosis factor for short disease-free survival $(\mathrm{P}=0.028)$.

Conclusion: Low CD44 expression was correlated with increased tumor recurrence and short disease-free survival, and low CD133 expression was associated with advanced tumor stage. We suggest that further studies be performed to evaluate whether the immunohistochemical method for determining the CD44 and the CD133 expressions is appropriate for exploring cancer stem-cell biology in patients with colorectal cancer.

Keywords: Colorectal neoplasms; CD40 antigens; CD133 antigen; Stem cell

\section{INTRODUCTION}

Colorectal cancer is the third most common cancer in men and the second most common cancer in women worldwide [1]. The

Received: May 7, 2015 - Accepted: June 6, 2015

Correspondence to: Seong Woo Hong, M.D.

Department of Surgery, Inje University Seoul Paik Hospital, 9 Mareunnae-ro, Jung-gu, Seoul 100-032, Korea

Tel: +82-2-2270-0021, Fax: +82-2-2270-0247

E-mail: hongsw@paik.ac.kr

(C) 2015 The Korean Society of Coloproctology

This is an open-access article distributed under the terms of the Creative Commons Attribution NonCommercial License (http://creativecommons.org/licenses/by-nc/3.0) which permits unrestricted noncommercial use, distribution, and reproduction in any medium, provided the original work is properly cited. incidence of colorectal cancer in East Asian countries, including Japan and Korea, has increased sharply, probably due to a Westernized diet and lifestyle [2]. Mortality from colorectal cancer accounts for $8 \%$ of all cancer deaths, and colorectal cancer is the fourth most common cause of death from cancer [1]. Recently, colorectal cancer mortality has decreased in developed countries owing to better treatments and early detection [3]. New chemotherapeutic agents and targeted therapies have shown promising results of improving survival in colorectal cancer patients $[4,5]$. However, more than $30 \%$ of stage III colon cancer patients suffer a recurrence even though they may have received a curative resection and adjuvant chemotherapy with oxaliplatin [4]. The median progression-free survival time in metastatic colorectal cancer patients is only 8.9 months even after treatment with cetuximab 
and chemotherapy [5]. Tumor recurrence and chemoresistance are the main problems that need to be solved if survival in cancer patients is to be prolonged.

Recently, cancer stem cells (CSCs) have received attention due to their role in cancer initiation, progression, and metastasis [6]. Their ability of self-renewal, unlimited proliferation, and multipotency are considered cancer stem-cell phenotypes, and they seem to be responsible for local relapse and metastasis by inducing resistance against traditional drug therapy [7]. Specific cell surface markers for CSCs are needed for identifying and sorting the CSCs. Several markers for CSCs have been investigated and proposed in colorectal cancer, and CD44 and CD133 have been the most frequently researched and are thought to be the most likely markers for colorectal CSCs [8-10]. In this study, we evaluated the expressions of CD44 and CD133 in colorectal cancer tissue by using the immunohistochemical staining method, and we analyzed the clinical significance of the results.

\section{METHODS}

\section{Patients and clinicopathological data}

One hundred sixty-two patients with a biopsy-proven colorectal adenocarcinoma who were operated on between January 1998 and August 2004 were enrolled in this study. Patients' data recorded in our colorectal cancer database were analyzed. The following clinicopathological factors were selected and evaluated: gender, age, location of tumor (right colon, left colon, or rectum), tumor size, tumor's gross appearance, carcinoembryonic antigen (CEA), carbohydrate antigen 19-9 (CA 19-9), TNM stage (American Joint Committee on Cancer. 7th ed.), tumor differentiation, and recurrence of tumor.

\section{Immunohistochemical staining method}

Staining for CD44 and CD133 was performed on primary colorectal cancer tissue, metastatic lymph nodes, and synchronous and metachronous metastatic tumor tissues if available. Tissue arrays were prepared by consigning them to the SuperBio Chips, Co. (Seoul, Korea). Tissue array blocks were sectioned to be $4 \mu \mathrm{m}$ in thickness, and immunohistochemical staining was performed using a Bond polymer detection kit and Bond-max autostainer (Leica Microsystems, Wetzlar, Germany) as follows: Deparaffinization by incubation in a dry oven at $60^{\circ} \mathrm{C}$ for 1 hour, antigen retrieval by using $\mathrm{pH} 6.0$ Bond epitope retrieval solution 1 at $100^{\circ} \mathrm{C}$ for 20 minutes, and peroxidase blocking with $0.3 \%$ hydrogen peroxide for 5 minutes. The samples were reacted with primary anti-CD44 antibody (DF1485, 1:200; Novocastra Laboratories, Newcastle upon Tyne, UK) or primary anti-CD133 antibody (AC133, 1:50; Miltenyi Biotec, Bergisch Gladbach, Germany) at room temperature for 15 minutes (CD44) and $30 \mathrm{~min}$ utes (CD133). Subsequently, tissues were incubated with polymer for 8 minutes and developed with 3, 3'-diaminobenzidine tetrahydrochloride-chromogen for 10 minutes and counter-stained with Mayer's hematoxylin. The negative control group was stained using an identical procedure, but omitting the primary antibody. Immunohistochemical staining of the specimens was evaluated by one pathologist (Y.K.K.).

The assessment of the CD44 and the CD133 expressions in the primary tumor was performed in the central and peripheral portions, and stronger expression results were accepted. The interpretation of the CD44 and the CD133 expressions was performed by a pathologist (Y.K.K.) who did not have clinical information on the patients. The stained tumor cell was scanned by using a high-power microscope and was scored according to the positivity of the tumor cells. The scores ranged from 0 to 3 for CD44 and from 0 to 2 for CD133.

\section{Statistical analysis}

Then CD44 and the CD133 expressions in the primary tumor vs. lymph nodes and in the primary tumor vs. metastatic tumor were compared by using the Wilcoxon signed rank test. The associations between clinicopathological factors and CD44 and CD133 expressions were analyzed by using the chi-square test. The Kaplan-Meier method was used to calculate the cumulative survival rate and to plot the survival curves. The log-rank test was used to compare the curves. A Cox proportional hazards regression was performed to confirm the prognostic value of the variables associated with survival. $\mathrm{P}<0.05$ was considered to be statistically significant.

\section{RESULTS}

The patients' median age was 61 years (range, $29-85$ years). The numbers of male and female patients were 90 and 72, respectively. The number of colon cancer cases was 88 , and the number of rectal cancer cases was 74 . Twenty-two cases were stage I, and 54, 70, and 16 cases were stages II, III, and IV, respectively. The mean follow-up period was 83 months (range, 2-172 months).

The CD44 and the CD133 expressions in the primary tumor, the metastatic lymph nodes, and the hepatic metastasis are shown in Fig. 1. The CD44 and the CD133 expression rates in the primary tumor, the metastatic lymph nodes, synchronous metastasis, and metachronous metastasis are presented in Table 1.

In 66 cases of paired primary tumor and metastatic lymph nodes, CD44 expression was stronger in the primary tumor than in the metastatic lymph nodes (primary $>$ lymph node in 37 cases, primary $=$ lymph node in 19 cases, primary $<$ lymph node in 10 cases, $\mathrm{P}<0.001)$. CD133 expression tended to be stronger in the primary tumor than in the metastatic lymph nodes (primary $>$ lymph node in 19 cases, primary $=$ lymph node in 39 cases, primary < lymph node in 8 cases, $\mathrm{P}=0.057$ ) (Table 2$)$.

In cases of paired primary and distant metastatic tumors (primary vs. synchronous metastasis in 8 cases and primary vs. metachronous metastasis in 15 cases), no differences in CD44 expression were observed $(\mathrm{P}=1.000$ and $\mathrm{P}=0.372$, respectively). Also, 


\section{$\begin{array}{cl}\text { Annals of } & \begin{array}{l}\text { Expression of the Cancer Stem Cell Mark } \\ \text { Immunohistochemical Staining Analysis }\end{array}\end{array}$}

\section{Coloproctology Injae Hong, et al.}
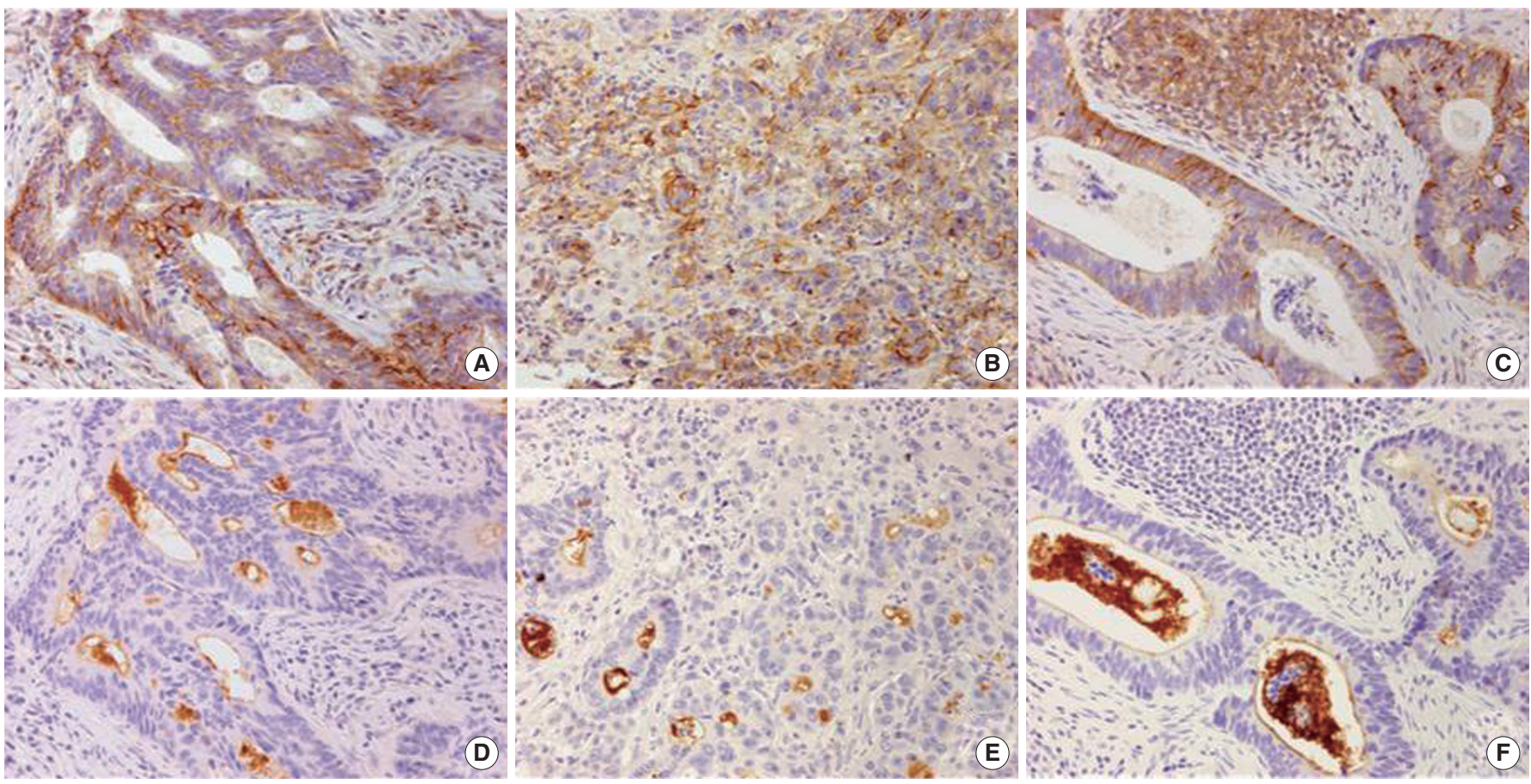

Fig. 1. Immunohistochemical staining of the expression of (A) CD44 in a primary colorectal adenocarcinoma $(\times 200)$, (B) CD44 in a metastatic liver tumor from a colorectal adenocarcinoma $(\times 200),(C)$ CD44 in a metastatic lymph node $(\times 200),(D)$ CD133 in a primary colorectal adenocarcinoma $(\times 200)$, (E) CD133 in a metastatic liver tumor from a colorectal adenocarcinoma $(\times 200)$, and $(F)$ CD133 in a metastatic lymph node $(\times 200)$.

Table 1. CD44 and CD133 expressions in tumor tissue

\begin{tabular}{|c|c|c|c|c|c|c|c|}
\hline \multirow{2}{*}{ Variable } & \multicolumn{4}{|c|}{ CD44 } & \multicolumn{3}{|c|}{ CD133 } \\
\hline & 0 & $1+$ & $2+$ & $3+$ & 0 & $1+$ & $2+$ \\
\hline Primary tumor & $22(13.6)$ & $42(25.9)$ & $46(28.4)$ & $52(32.1)$ & $79(48.8)$ & $60(37.0)$ & $23(14.2)$ \\
\hline Lymph node & $29(43.9)$ & $22(33.3)$ & $11(16.7)$ & $4(2.5)$ & $43(65.2)$ & $18(27.3)$ & $5(7.6)$ \\
\hline Synchronous metastasis & - & $3(37.5)$ & $4(50.0)$ & $1(12.5)$ & $3(37.5)$ & $5(62.5)$ & - \\
\hline Metachronous metastasis & $3(20.0)$ & $3(20.0)$ & $5(33.3)$ & $4(26.7)$ & $5(33.3)$ & $4(26.7)$ & $6(40.0)$ \\
\hline
\end{tabular}

Values are presented as number (\%).

Table 2. Comparison of CD 44 and CD133 expressions in cases of paired primary tumors and metastatic lymph nodes (66 cases)

\begin{tabular}{lcccc}
\hline & Primary $^{\mathrm{a}}>$ LN & Primary $=$ LN & Primary $<$ LN & P-value \\
\hline CD44 & $37(56.1)$ & $19(28.8)$ & $10(15.2)$ & $<0.001$ \\
CD133 & $19(28.8)$ & $39(59.1)$ & $8(12.1)$ & 0.057 \\
\hline
\end{tabular}

Values are presented as number (\%).

$\mathrm{LN}$, metastatic lymph nodes.

aprimary tumor.

no differences in CD133 expression were noted between the primary tumor and a synchronous or metachronous metastasis $(\mathrm{P}=$ 0.655 and $\mathrm{P}=0.157$, respectively).

No significant correlation was noted between CD44 expression and $\mathrm{CD} 133$ expression in the primary tumor $(\mathrm{P}=0.466)$ (Fig. 2$)$.
The relationships between CD44 and CD133 expressions in the primary tumor and the other clinicopathological characteristics were analyzed. The patients in which recurrence developed during the follow-up period showed low expression rates of CD44 (P $=0.017)$. In rectal cancer, the CD133 expression rate was higher than that it was in colon cancer $(\mathrm{P}=0.017)$. The $\mathrm{CD} 133$ expression rate was lower in cases with elevated CA 19-9 serum levels ( $\mathrm{P}$ $=0.028)$ and advanced $\mathrm{T}$ stage $(\mathrm{P}=0.038)$ (Table 3$)$. No significant correlation were noted between other clinicopathological factors, including gender, age, tumor size, gross appearance of tumor, CEA, TNM stage, and histology (differentiation of tumor), and the $\mathrm{CD} 44$ and the $\mathrm{CD} 133$ expressions in the primary tumor.

The overall survival and the disease-free survival according to CD44 and CD133 expressions were evaluated by using the Kaplan-Meier method (Fig. 3). The disease-free survival in patients 


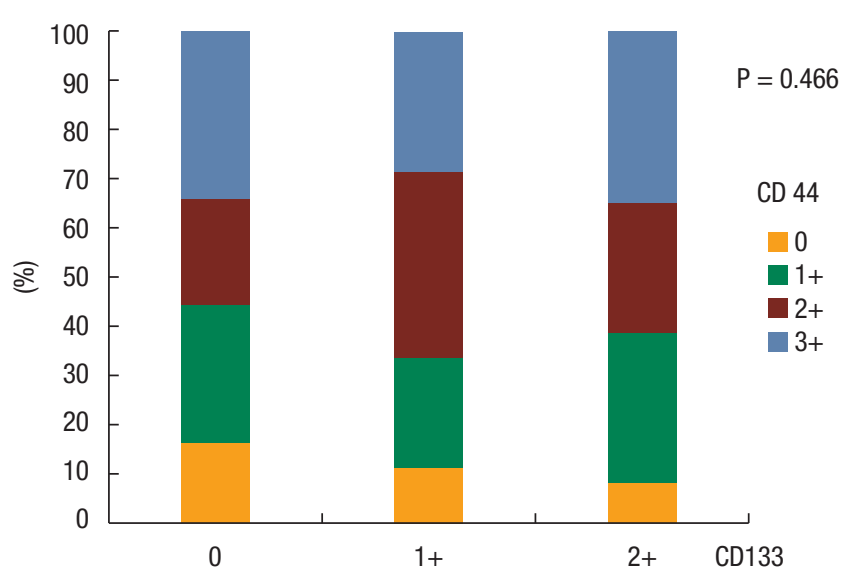

Fig. 2. Relationship between CD44 and CD133 expressions in a colorectal adenocarcinoma.

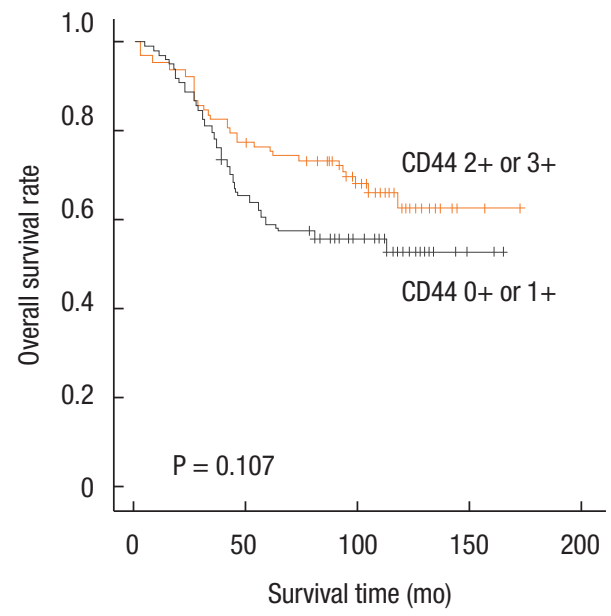

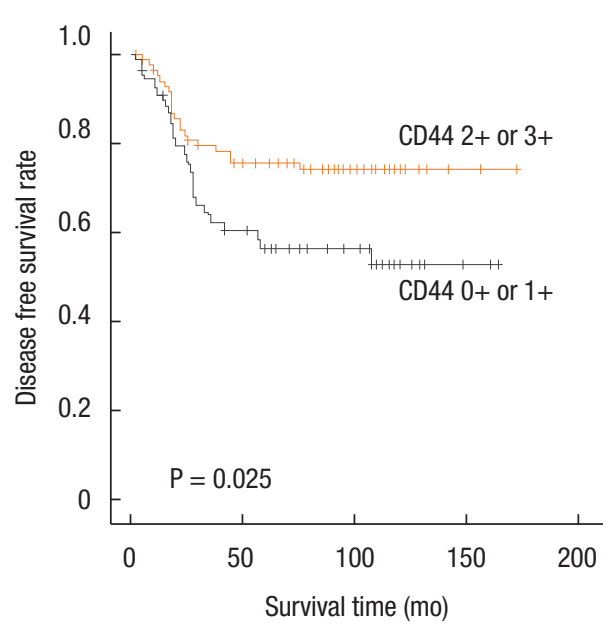

C

(A) with a high CD44-expressing tumor was significantly better than that in patients with a low CD44-expressing tumor $(\mathrm{P}=0.025)$. No significant correlations were found between the overall survival time and the CD44 and the CD133 expression, and between the disease-free survival time and the CD133 expression ( $\mathrm{P}=$ $0.107, \mathrm{P}=0.206$, and $\mathrm{P}=0.216$, respectively). A multivariate analysis was performed to verify whether CD44 expression was an independent prognostic factor for disease-free survival in colorectal cancer patients (Table 4). CD44 expression, gross appearance of the tumor, CA 19-9, and TNM stage were proven to be independent prognostic factors for disease-free survival in colorectal cancer patients.

\section{DISCUSSION}

Currently, CSCs are considered to be responsible for tumor initiation, progression, and metastasis [6]. The major portion of the

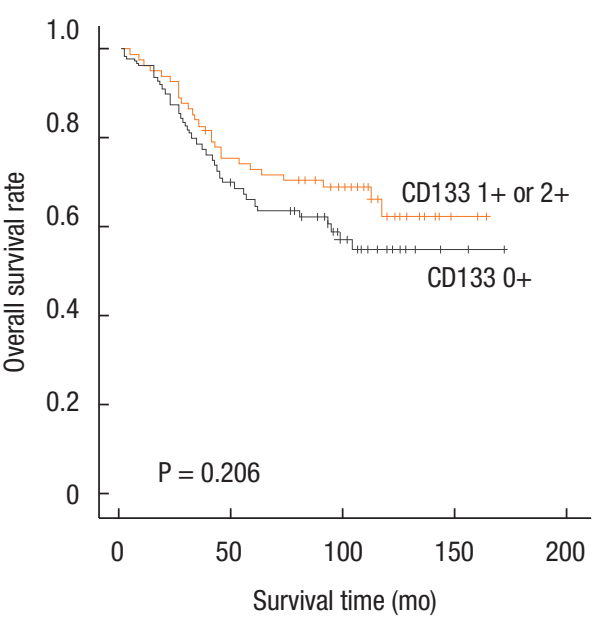

B

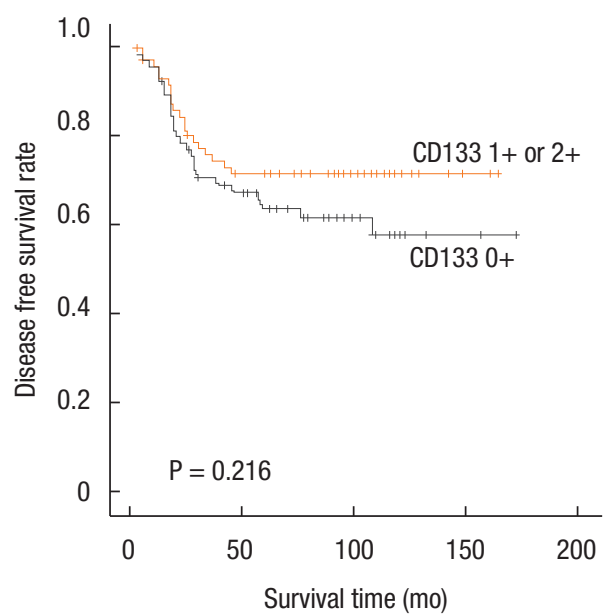

D

Fig. 3. Overall survival rate as a function of the survival time for CD44 (A) and CD133 (B) expressions, and disease-free survival rate as a function of survival time for CD44 (C) and CD 133 (D) expressions. 
Table 3. Relationship between CD44 and CD133 expressions in the primary tumor and clinicopathological factors

\begin{tabular}{|c|c|c|c|c|c|c|}
\hline \multirow{2}{*}{ Variable } & \multicolumn{2}{|c|}{ CD44 expression } & \multirow{2}{*}{ P-value } & \multicolumn{2}{|c|}{ CD133 expression } & \multirow{2}{*}{ P-value } \\
\hline & 0 or $1+$ & $2+$ or $3+$ & & 0 & $1+$ or $2+$ & \\
\hline Gender & & & 0.886 & & & 0.419 \\
\hline Male & $36(40.0)$ & $54(60.0)$ & & $47(52.2)$ & $43(47.8)$ & \\
\hline Female & $28(38.9)$ & $44(61.1)$ & & $33(45.8)$ & 39 (54.2) & \\
\hline Age (yr) & & & 0.690 & & & 0.626 \\
\hline$\leq 62$ & $35(40.9)$ & $52(59.1)$ & & $45(51.1)$ & $43(48.9)$ & \\
\hline$>62$ & $28(37.8)$ & $46(62.2)$ & & $35(47.3)$ & $39(52.7)$ & \\
\hline Tumor location & & & 0.569 & & & 0.017 \\
\hline Colon & $33(37.5)$ & $55(62.5)$ & & $51(58.0)$ & $37(42.0)$ & \\
\hline Rectum & $31(41.9)$ & $43(58.1)$ & & $29(39.2)$ & $45(60.8)$ & \\
\hline Tumor size (cm) & & & 0.893 & & & 0.349 \\
\hline$\leq 5.0$ & $34(40.0)$ & $51(60.0)$ & & $39(45.9)$ & $46(54.1)$ & \\
\hline$>5.0$ & $30(39.0)$ & $47(61.0)$ & & $41(53.2)$ & $36(46.8)$ & \\
\hline Gross appearance & & & 0.413 & & & 0.413 \\
\hline Fungating & $46(37.7)$ & $76(62.3)$ & & $58(47.5)$ & $64(52.5)$ & \\
\hline Ulcerating & $18(45.0)$ & $22(55.0)$ & & $22(55.0)$ & $18(45.0)$ & \\
\hline CEA (ng/mL) & & & 0.725 & & & 0.821 \\
\hline$\leq 5$ & $45(38.8)$ & $71(61.2)$ & & $59(50.9)$ & $57(49.1)$ & \\
\hline$>5$ & $18(41.9)$ & $25(58.1)$ & & $21(48.8)$ & $22(51.2)$ & \\
\hline CA 19-9 (U/mL) & & & 0.411 & & & 0.028 \\
\hline$\leq 37$ & $55(38.7)$ & 87 ( (61.3) & & $68(47.9)$ & $74(52.1)$ & \\
\hline$>37$ & $7(50.0)$ & 7 (50.0) & & $11(78.6)$ & $3(21.4)$ & \\
\hline T stage & & & 0.578 & & & 0.038 \\
\hline T1 or T2 & $9(34.6)$ & $17(65.4)$ & & $8(30.8)$ & 18 (69.2) & \\
\hline T3 or T4 & $55(40.4)$ & $81(59.6)$ & & $72(52.9)$ & $64(47.1)$ & \\
\hline TNM stage & & & 0.711 & & & 0.334 \\
\hline I & $7(31.8)$ & $15(68.2)$ & & $7(31.8)$ & $15(68.2)$ & \\
\hline$\|$ & $20(37.0)$ & $34(63.0)$ & & $27(50.0)$ & $27(50.0)$ & \\
\hline III & $31(44.3)$ & $39(55.7)$ & & $38(54.3)$ & $32(45.7)$ & \\
\hline IV & $6(37.5)$ & $10(62.5)$ & & $8(50.0)$ & $8(50.0)$ & \\
\hline Histology ${ }^{a}$ & & & 0.144 & & & 0.533 \\
\hline Well differentiated & $9(47.4)$ & $10(52.6)$ & & $11(57.9)$ & $8(42.1)$ & \\
\hline Moderately differentiated & $51(41.8)$ & $72(58.5)$ & & $61(49.6)$ & $62(50.4)$ & \\
\hline Poorly differentiated & $4(20.0)$ & $16(80.0)$ & & $8(40.0)$ & $12(60)$ & \\
\hline Recurrence & & & 0.017 & & & 0.247 \\
\hline Absent & $27(31.0)$ & $60(69.0)$ & & $40(46.0)$ & $47(54.0)$ & \\
\hline Present & $24(52.2)$ & $22(47.8)$ & & $26(56.5)$ & $20(43.5)$ & \\
\hline
\end{tabular}

Values are presented as number (\%).

CEA, carcinoembryonic antigen; CA 19-9, carbohydrate antigen 19-9.

aDifferentiation of tumor.

cancer mass is differentiated, rapidly growing, and susceptible to traditional therapy such as chemotherapy or radiotherapy, but a very minor portion of the tumor mass is comprised of CSCs, which are slow growing and more resistant to chemo- and radiotherapy. After chemo- or radiotherapy induces tumor shrinkage,
CSCs can survive and differentiate into a rapid-growing phenotype with tumor-forming potential. Therefore, CSCs are suspected to be the main cause of tumor relapse and cancer mortality [11]. Targeted therapy directed toward CSCs may be useful in cancer therapy for reducing future relapses. 
Table 4. Univariate and multivariate analyses of disease-free survival in colorectal cancer patients

\begin{tabular}{|c|c|c|c|c|c|c|}
\hline \multirow{2}{*}{ Variable } & \multicolumn{3}{|c|}{ Univariate } & \multicolumn{3}{|c|}{ Multivariate } \\
\hline & HR & $95 \% \mathrm{Cl}$ & P-value & $\mathrm{HR}$ & $95 \% \mathrm{Cl}$ & P-value \\
\hline Tumor location, rectum & 1.049 & $0.584-1.882$ & 0.873 & - & - & - \\
\hline \multicolumn{7}{|l|}{ Size } \\
\hline$>5 \mathrm{~cm}$ & 0.686 & $0.375-1.253$ & 0.220 & - & - & - \\
\hline \multicolumn{7}{|l|}{ Gross appearance } \\
\hline Infiltrative & 2.752 & $1.513-5.004$ & 0.001 & 2.571 & $1.295-5.107$ & 0.007 \\
\hline \multicolumn{7}{|l|}{ CEA } \\
\hline$>5 \mathrm{ng} / \mathrm{mL}$ & 1.689 & $0.908-3.139$ & 0.098 & 1.422 & $0.730-2.770$ & 0.301 \\
\hline \multicolumn{7}{|l|}{ CA 19-9 } \\
\hline$>37 \mathrm{U} / \mathrm{mL}$ & 4.152 & $1.748-9.864$ & 0.001 & 3.699 & $1.486-9.209$ & 0.005 \\
\hline \multicolumn{7}{|l|}{ TNM stage } \\
\hline III or IV & 11.225 & $4.421-28.498$ & $<0.001$ & 10.199 & $3.911-26.595$ & $<0.001$ \\
\hline \multicolumn{7}{|l|}{ Histology } \\
\hline Poorly differentiated & 3.413 & $1.683-6.923$ & 0.001 & 1.734 & $0.775-3,876$ & 0.180 \\
\hline \multicolumn{7}{|l|}{ CD44 } \\
\hline $2+$ or $3+$ & 0.519 & $0.289-0.932$ & 0.028 & 0.505 & $0.274-0.931$ & 0.028 \\
\hline \multicolumn{7}{|l|}{ CD133 } \\
\hline $1+$ or $2+$ & 0.693 & $0.385-1.247$ & 0.221 & 0.944 & $0.506-1.761$ & 0.856 \\
\hline
\end{tabular}

$\mathrm{HR}$, hazard ratio; $\mathrm{Cl}$, confidence interval; CEA, carcinoembryonic antigen; CA 19-9, carbohydrate antigen 19-9.

Useful surface markers for CSCs are very much needed for research and treatment of various cancers. In colorectal cancer, CD44 and CD133 have been intensively evaluated [8-10]. Dalerba et al. [8] reported that a minority subpopulation of EpCAM ${ }^{\text {high }}$ / CD44 positive cells could initiate tumor formation with a small number of colorectal cancer cells. Tumors that originated from $\mathrm{EpCAM}^{\text {high }} / \mathrm{CD} 44$ positive cells reproduced the full morphological and phenotypic heterogeneity of their parental lesions. The authors concluded that CD44 could be one of the stem cell markers for colorectal cancer [8]. In 2007, two separate research groups reported that CD133-expressing cancer cells could initiate tumor growth, but CD133 negative cells could not. They concluded that CD133 positive cells might be the CSCs in colon cancer $[9,10]$. Therefore, in this study, we aimed to assess the expressions of CD44 and CD133 in colorectal cancer tissue by using immunohistochemical staining and to analyze the clinical significance of those expressions related to other clinicopathological data and survival results.

In 66 cases of paired primary tumor and metastatic lymph nodes, CD44 expression was stronger in the primary tumor than in metastatic lymph nodes $(\mathrm{P}<0.001)$, and CD133 expression tended to be stronger in the primary tumor than in the metastatic lymph nodes $(P=0.057)$. In cases of primary and distant metastatic tumors, no significant difference was observed in this study. Before this study, we conjectured that the metastatic tumor should have a stronger expression than the primary tumor because the metastasis might be caused by a small portion of the tumor cells that had stem-cell biology; therefore, we hypothesized that the metastatic tumor might have a greater population of CSCs. However, the results were contrary to our expectations. We suggest that these results should be confirmed by using other laboratory methods. In contrast to our results, Puglisi et al. [12] used flow cytometry to show that CD133 expression was higher in metastatic liver tumors than in primary colorectal cancer tumors.

No correlation between the expressions of CD44 and CD133 in cancer tissue was found in this study. Du et al. [13] reported that CD44 and CD133 double positive cells were extremely rare in their study. Galizia et al. [14] also showed that CD133 expression was not correlated with CD44 expression in colorectal cancer tissues. These results suggest that neither CD44 nor CD133 may be a strong, specific marker for CSCs in the immunohistochemical staining method.

Some controversy exists over the correlations between CD44 and CD133 expressions and other clinicopathological factors. In our study, CD44 expression was lower in cases that developed recurrence during the follow-up period $(\mathrm{P}=0.017)$. The other clinicopathological factors were not correlated with CD44 expression. Pitule et al. [15] reported that cases with low CD44 expression had a high incidence of lymph node metastasis. On the contrary, other studies showed that high CD44 expression was related to advanced stage, poor differentiation [16], depth of invasion, and lymph node involvement [17]. In this study, CD133 expression 
was lower in colon cancer $(\mathrm{P}=0.017)$ than in rectal cancer and in cases with elevated CA 19-9 serum levels $(\mathrm{P}=0.028)$ and advanced $\mathrm{T}$ stage $(\mathrm{P}=0.038)$. However, other researchers reported that high CD133 expression was related to advanced T stage [18] and to frequent node and distant metastasis [19]. One pooled study showed that CD133 was not significantly associated with the depth of invasion, tumor differentiation, lymph node metastasis, or lymphatic invasion [20].

Some controversy also exists over the relationships between CD44 and CD133 expressions and the prognosis for colorectal cancer patients. In our study, low CD44 expression was identified as an independent prognostic factor for shorter disease-free survival. Similarly, two studies, which evaluated the expression of CD44 by using immunohistochemical staining, demonstrated that loss of CD44 expression was related to a worse prognosis for colorectal cancer patients $[21,22]$. In contrast, Huh et al. [17] reported that CD44 expression was an independent unfavorable prognostic factor for overall survival. Another two studies showed that CD44 expression was not significantly associated with survival $[15,18]$. In this study, CD133 was not significantly associated with the survival interval. Several studies showed that CD133 expression predicted a poor prognosis for colorectal cancer patients $[23,24]$. On the contrary, Pitule et al. [15] showed that patients with high CD133 expression had longer a disease-free interval. Other studies reported that CD133 was not significantly correlated with the survival time [18, 22, 25].

In conclusion, in this study, low CD44 expression was correlated with increased tumor recurrence and shorter disease-free survival, and low CD133 expression was associated with advanced tumor stage. We suggest that further studies be performed to evaluate whether the immunohistochemical method for determining the CD44 and the CD133 expressions is appropriate for exploring cancer stem-cell biology in patients with colorectal cancer.

\section{CONFLICT OF INTEREST}

No potential conflict of interest relevant to this article was reported.

\section{ACKNOWLEDGMENTS}

This work was supported by a grant from Inje University, 2011.

\section{REFERENCES}

1. Ferlay J, Shin HR, Bray F, Forman D, Mathers C, Parkin DM. Estimates of worldwide burden of cancer in 2008: GLOBOCAN 2008. Int J Cancer 2010;127:2893-917.

2. Jemal A, Center MM, DeSantis C, Ward EM. Global patterns of cancer incidence and mortality rates and trends. Cancer Epidemiol Biomarkers Prev 2010;19:1893-907.
3. Center MM, Jemal A, Smith RA, Ward E. Worldwide variations in colorectal cancer. CA Cancer J Clin 2009;59:366-78.

4. Andre T, Boni C, Navarro M, Tabernero J, Hickish T, Topham C, et al. Improved overall survival with oxaliplatin, fluorouracil, and leucovorin as adjuvant treatment in stage II or III colon cancer in the MOSAIC trial. J Clin Oncol 2009;27:3109-16.

5. Van Cutsem E, Kohne CH, Hitre E, Zaluski J, Chang Chien CR, Makhson A, et al. Cetuximab and chemotherapy as initial treatment for metastatic colorectal cancer. N Engl J Med 2009;360: 1408-17.

6. Chen S, Huang EH. The colon cancer stem cell microenvironment holds keys to future cancer therapy. J Gastrointest Surg 2014; 18:1040-8.

7. Gangemi R, Paleari L, Orengo AM, Cesario A, Chessa L, Ferrini S, et al. Cancer stem cells: a new paradigm for understanding tumor growth and progression and drug resistance. Curr Med Chem 2009; 16:1688-703.

8. Dalerba P, Dylla SJ, Park IK, Liu R, Wang X, Cho RW, et al. Phenotypic characterization of human colorectal cancer stem cells. Proc Natl Acad Sci U S A 2007;104:10158-63.

9. Ricci-Vitiani L, Lombardi DG, Pilozzi E, Biffoni M, Todaro M, Peschle C, et al. Identification and expansion of human coloncancer-initiating cells. Nature 2007;445:111-5.

10. O’Brien CA, Pollett A, Gallinger S, Dick JE. A human colon cancer cell capable of initiating tumour growth in immunodeficient mice. Nature 2007;445:106-10.

11. Morrison R, Schleicher SM, Sun Y, Niermann KJ, Kim S, Spratt $\mathrm{DE}$, et al. Targeting the mechanisms of resistance to chemotherapy and radiotherapy with the cancer stem cell hypothesis. J Oncol 2011;2011:941876.

12. Puglisi MA, Sgambato A, Saulnier N, Rafanelli F, Barba M, Boninsegna $\mathrm{A}$, et al. Isolation and characterization of CD133+ cell population within human primary and metastatic colon cancer. Eur Rev Med Pharmacol Sci 2009;13 Suppl 1:55-62.

13. Du L, Wang H, He L, Zhang J, Ni B, Wang X, et al. CD44 is of functional importance for colorectal cancer stem cells. Clin Cancer Res 2008;14:6751-60.

14. Galizia G, Gemei M, Del Vecchio L, Zamboli A, Di Noto R, Mirabelli $\mathrm{P}$, et al. Combined CD133/CD44 expression as a prognostic indicator of disease-free survival in patients with colorectal cancer. Arch Surg 2012;147:18-24.

15. Pitule P, Cedikova M, Daum O, Vojtisek J, Vycital O, Hosek P, et al. Immunohistochemical detection of cancer stem cell related markers CD44 and CD133 in metastatic colorectal cancer patients. Biomed Res Int 2014;2014:432139.

16. Zavrides HN, Zizi-Sermpetzoglou A, Panousopoulos D, Athanasas G, Elemenoglou I, Peros G. Prognostic evaluation of CD44 expression in correlation with bcl-2 and p53 in colorectal cancer. Folia Histochem Cytobiol 2005;43:31-6.

17. Huh JW, Kim HR, Kim YJ, Lee JH, Park YS, Cho SH, et al. Expression of standard CD44 in human colorectal carcinoma: association with prognosis. Pathol Int 2009;59:241-6. 
18. Choi D, Lee HW, Hur KY, Kim JJ, Park GS, Jang SH, et al. Cancer stem cell markers CD133 and CD24 correlate with invasiveness and differentiation in colorectal adenocarcinoma. World J Gastroenterol 2009;15:2258-64.

19. Horst D, Scheel SK, Liebmann S, Neumann J, Maatz S, Kirchner T, et al. The cancer stem cell marker CD133 has high prognostic impact but unknown functional relevance for the metastasis of human colon cancer. J Pathol 2009;219:427-34.

20. Wang K, Xu J, Zhang J, Huang J. Prognostic role of CD133 expression in colorectal cancer: a meta-analysis. BMC Cancer 2012; 12:573.

21. Ngan CY, Yamamoto H, Seshimo I, Ezumi K, Terayama M, Hem$\mathrm{mi} \mathrm{H}$, et al. A multivariate analysis of adhesion molecules expression in assessment of colorectal cancer. J Surg Oncol 2007;95:65262.
22. Lugli A, Iezzi G, Hostettler I, Muraro MG, Mele V, Tornillo L, et al. Prognostic impact of the expression of putative cancer stem cell markers CD133, CD166, CD44s, EpCAM, and ALDH1 in colorectal cancer. Br J Cancer 2010;103:382-90.

23. Horst D, Kriegl L, Engel J, Kirchner T, Jung A. CD133 expression is an independent prognostic marker for low survival in colorectal cancer. Br J Cancer 2008;99:1285-9.

24. Coco C, Zannoni GF, Caredda E, Sioletic S, Boninsegna A, Migaldi $\mathrm{M}$, et al. Increased expression of CD133 and reduced dystroglycan expression are strong predictors of poor outcome in colon cancer patients. J Exp Clin Cancer Res 2012;31:71.

25. Zhou F, Mu YD, Liang J, Liu ZX, Chen HS, Zhang JF. Expression and prognostic value of tumor stem cell markers ALDH1 and CD133 in colorectal carcinoma. Oncol Lett 2014;7:507-12. 